\title{
PKC Mediates PACSIN2 to Regulate Hypertrophic Cardiomyocytes in the Aggravation of the Compensatory Function
}

Siang Wei ( $\nabla$ weisiang27@foxmail.com )

Fudan University https://orcid.org/0000-0002-1162-7306

\section{Ran Xu}

Fudan University

Xiaohui Li

Fudan University

\section{Zhiwen Ding}

Fudan University

Yunzeng Zou

Fudan University

Research article

Keywords: PKC, PACSIN2, positive regulation, myocardial contraction,myocardial hypertrophy

Posted Date: September 30th, 2021

DOl: https://doi.org/10.21203/rs.3.rs-868336/v1

License: (9) This work is licensed under a Creative Commons Attribution 4.0 International License. Read Full License 


\section{Abstract}

Background Myocardial hypertrophy ( $\mathrm{MH})$ is an independent risk factor for cardiovascular and cerebrovascular events in patients with hypertension and myocardial infarction. Recent studies have shown that PACSIN2 is associated with cardiovascular disease; however, the function of cardiomyocytes remains unclear. Here, the interaction between protein kinase $C$ (PKC) and PACSIN2 and the manner in which PACSIN2 regulates $\mathrm{MH}$ was studied.

Methods The cell models were constructed with angiotensin II (AII) and PMA; qRT-PCR and Western Blot were used to verify the relative protein expression of PKC and PACSIN2; immunofluorescence was used to determine the effect of PKC upregulation on the morphology of hypertrophic cardiomyocytes after an overexpression and silencing of PACSIN2 by qRT-PCR and Western blot analysis.

Results The results indicate that PKC positively regulates the expression of PACSIN2 and causes MH phenotype. The overexpression of PACSIN2 was not solely responsible for $\mathrm{MH}$, while the overexpression of PACSIN2 under MH caused hypertrophy to increase. In addition, the high expression of PACSIN2 caused an increase in a-MHC mRNA, but it was not related to the expression of tubulin. The morphological results indicate that the overexpression of $\mathrm{PKC}$ causes $\mathrm{MH}$, which causes heart failure.

Conclusion Studies have shown that PACSIN2 plays an important role in maintaining myocardial cell contraction and $\mathrm{MH}$. To clarify, the PAC interacting protein and the cardiomyocyte function provide a theoretical basis for the pathology and treatment of hypertrophic cardiomyopathy.

\section{Introduction}

Myocardial hypertrophy $(\mathrm{MH})$ is a kind of ventricular wall thickening, which acts as the main feature that can induce cardiac systolic and diastolic dysfunction for a long time and, eventually, lead to functional changes due to myocardial failure, arrhythmia, myocardial ischemia and even sudden death ${ }^{1-3}$. MH is closely related to a variety of cardiovascular diseases. Studies have shown that $\mathrm{MH}$ is a common intermediate link between heart failure and hypertension, coronary heart disease, myocardial infarction and other cardiovascular diseases (CVD) ${ }^{4-6}$. MH can be categorized into physiological $\mathrm{MH}$ and pathological $\mathrm{MH}$. The two types of hypertrophies initially developed into an adaptive response to cardiac stress; however, there are significant differences in the underlying molecular mechanism, cardiac phenotype and prognosis ${ }^{7}$. Over time, physiological hypertrophy maintains heart function, whereas pathological hypertrophy is accompanied by adverse cardiovascular events, including heart failure, arrhythmia and death. Physiological MH refers to compensatory hypertrophy caused by pregnancy, physical exercise and so on. This type of hypertrophy can be self-recovered. It is characterized by increase in myocardial cell volume and myocardial mass, cytoskeleton reorganization, transcription factor activation and enhanced protein synthesis; pathological $\mathrm{MH}$ is the heart's compensatory response to various stimuli, such as overload, valvular insufficiency, hypertension, ischemic heart disease, hereditary cardiomyopathy and so on. The compensatory response of the heart to long-term overload is 
irreversible ${ }^{8}$. Long-term pathological hypertrophy can induce cardiac systolic and diastolic insufficiency, and it can ultimately lead to myocardial failure, arrhythmia, myocardial ischemia and even sudden death $^{9}$. The signal transmission in cardiomyocytes and the interaction with other types of heart cells (such as cardiac fibroblasts, vascular endothelial cells, and vascular smooth muscle cells) are extremely complex. In addition, the specific/selective contribution of different signal cascades to compensatory heart growth and the transition to decompensated heart growth and heart failure require further research ${ }^{10}$. The transduction signal pathways activated by various stimuli are quite different, and numerous complex signal pathways play a vital role in regulating the central pathway and effector of cardiac growth response.

PACSIN2, also known as glutinin, includes the Bin-AmiphisinRvs (BAR-) protein superfamily, which mediates the generation of carriers during the process of intracellular transport and/or regulates the membrane deformation that is required for the transport of specific cargo proteins ${ }^{11}$. Among the three family members, called PACSIN1, PACSIN2 and PACSIN3, PACSIN2 is widely distributed, while PACSIN1 is mainly expressed in the neuronal tissue ${ }^{12}$, and PACSIN3 is limited to muscle, heart and lungs. Current research shows that PACSIN2 is mainly associated with leukemia, diabetes, kidney disease and hepatitis. PACSIN2 polymorphism is associated with thiopurine-induced hematological toxicity in children with acute lymphoblastic leukemia undergoing maintenance therapy ${ }^{13}$. PACSIN2 accelerates nephrin trafficking and is upregulated in diabetic kidney disease ${ }^{14}$. Two recent studies have clarified the role of PACSIN2 in endocytosis unrelated to caverns ${ }^{15,16}$. PACSIN2 directly binds to cave protein 1 through its FBAR domain and is thought to recruit generators to the cave protein separation site ${ }^{14}$. In addition to its location in vesicles, PACSIN2 is also found in tubular structures ${ }^{17,18}$. It expresses the phenotype of PACSIN2 KO mice and provides evidence that through its significance in promoting apical endocytosis, this molecule plays a role in controlling microvillar morphology ${ }^{19}$. PACSIN2 plays an important part in the physiological and pathological process of human health; however, the manner of playing the dynamic mechanism remains to be studied.

Previous studies on PACSIN2 focused on the functional characteristics of other tissues and cells. However, the function of PACSIN2 in cardio myogenesis has not been studied, and there is no information regarding the exact role of the protein in cardiac function and the biological network. This study mainly explored the involvement of protein kinase C (PKC)-mediated PACSIN2 in the development and progression of hypertrophic cardiomyopathy. This study provides insights into the function of PACSIN2 in cardiomyocytes, and it may present a potential therapeutic target for the treatment of cardiomyopathy.

Therefore, in the present work, cardiomyocytes overexpressing and silencing PACSIN2 were used to analyze the function of PACSIN2 in normal and hypertrophic states. The results of the study provide important insights for the study of PACSIN2 in regulating and maintaining the normal function of the heart and the physiological and pathological functions of hypertrophic cardiomyopathy.

\section{Materials And Methods}




\subsection{Mouse preparation}

In this study, male SD rats of 8-10 g weight and 1-3 days of age were raised in the Animal Breeding Center of Beijing Viewsolid Biotech Co., Ltd. [SYXK (Beijing) 2016-0039., SPF-level management of the Experimental Animal Center. The experimental animals were provided by Beijing Viewsolid Biotech Co., Ltd [SCXK (Beijing) 2016-0009.. All experimental mice were cared for in accordance with the 3R principle. The approval number of the Experimental Animal Ethics Committee is 20200037.

\subsection{Primary cardiomyocyte culture}

After the suckling mice were immersed in $75 \%$ alcohol for disinfection, their hearts were immediately opened in an ultra-clean workbench in order to be extracted and washed twice in a glass dish filled with ice PBS solution ( $4{ }^{\circ} \mathrm{C}$ PBS). Following this, the hearts were transferred to a $10 \mathrm{ml}$ sterile centrifuge tube, and digestive enzyme (containing $0.1 \%$ trypsin) prepared with PBS solution for digestion was added. The entire digestion process was carried out under constant temperature and stirring at $37^{\circ} \mathrm{C}, 130 \mathrm{rpm} / \mathrm{min}$. After every 8 min of digestion, the supernatant was sucked in the centrifuge tube, it was added to the same amount of DMEM-F12 culture medium containing 10\% FBS and a pipette was used to evenly mix it. This process was repeated approximately 7-8 times until the tissue block was completely digested, the collected tubes were centrifuged at $1000 \mathrm{rpm}$ for $6 \mathrm{~min}$ at room temperature, the supernatant was discarded, the cells were resuspended in a DMEM medium containing 10\% FBS the cardiomyocytes obtained each time were combined in a petri dish and statically incubated in an incubator containing $5 \%$ $\mathrm{CO} 2$ at $37^{\circ} \mathrm{C}$. The morphology, beating frequency, rhythm and intensity of the cardiomyocytes were observed under an electron microscope following the culturing in an incubator containing $5 \% \mathrm{CO} 2$ at $37^{\circ} \mathrm{C}$ for 24 hours, and their growth status was gauged.

\subsection{HL1 cardiomyocyte culture}

The cells were cultured in a DMEM-F12 medium containing 10\% fetal bovine serum. When the myocardial cell density grew to $70-80 \%$, the cells were transferred out of the culture according to $1: 4$.

\subsection{Construction of $\mathrm{MH}$ cell model}

When the myocardial cell density grows to $60-70 \%$, the cell culture medium is changed to a serum-free DMEM-F12 one, starved for $12 \mathrm{~h} .1 \times 10^{-\wedge} 6 \mathrm{mmol}$ angiotensin (All) is added, and the stimulation time is set to $0 \mathrm{~h}, 6 \mathrm{~h}, 12 \mathrm{~h}, 24 \mathrm{~h}$ and $48 \mathrm{~h}$. The mRNA expressions of ANP, BNP and PACSIN2 were detected to confirm the success of the cell model.

\subsection{Cellular drug treatment}


In order to achieve high expression of PKC in cardiomyocytes, the PKC agonist drug PMA is used. The concentration of the drug added to the cells is based on the amount provided in the instructions. The expression of $12 \mathrm{~h}$ ANP, BNP and PACSIN2 mRNA and the expression of PKC, PACSIN2 and RCAN1.4 protein are detected.

\subsection{Plasmid construction and transfection}

A PACSIN2 shRNA (sh-PACSIN2) plasmid was constructed, and the design and construction were completed by Beijing Viewsolid Biotech Co., Ltd. The purpose of constructing siRNA was to downregulate the expression of PACSIN2. According to the manufacturer's instructions, polybrene (viral transfer aid) is diluted at the ratio of transfer aid: virus $=1: 500$, and $500 \mu$ medium is added $2 \mathrm{~h}$ after adding the cells.

\subsection{Cellular immunofluorescence histochemistry}

The cells were diluted in multiples in a 96 -well plate (2000 is the most effective). They were fixed with cold $4 \%$ paraformaldehyde for 30 min, washed 3 times with PBS for 5 min each time, permeated with $0.3 \%$ Trition for 5 min (diluted with PBS), washed 3 times with PBS for 5 min each time. They were blocked with $5 \%$ goat serum (PBS) on a room temperature shaker for $1 \mathrm{~h}$; the primary antibody was incubated overnight at $4{ }^{\circ} \mathrm{C}$ : $\mathrm{a}$-actin (1:50); then, the primary antibody was recovered and washed 3 times with PBS for 5 min each; the PBS (1:1000) Dilution was used; the absorbance of the rabbit secondary antibody was $488 \mathrm{~nm}$. This was incubated in an oven at $37^{\circ} \mathrm{C}$ in the dark for $1 \mathrm{~h}$; it was washed with PBS 3 times, for 5 min each time, and was observed under a microscope.

\subsection{Quantitative reverse transcription polymerase chain reaction}

The total RNA from primary cardiomyocytes and HL1 cells were extracted using Trizol reagent (Invitrogen) according to the manufacturer's protocol for a-MHC and $\beta-M H C$ mRNA expression; $1 \mathrm{mg}$ of total RNA was reverse-transcribed into cDNA using PrimeScript ${ }^{\mathrm{T} M}$ RTMaster Mix (TaKaRa, Dalian, China). Then, real-time PCR was performed using a SYBR ® Premix Ex TaqTM II kit (TakaRa) on the 7500 Fast Real-Time PCR System (Applied Biosystems, Foster City, CA, USA).

\subsection{West Blotting}

The total protein was extracted using a RIPA lysis buffer (lifetime) for 20 min on ice. Its concentration was determined with a BCA protein assay kit (general, Shanghai, China). Equal amounts of protein were separated by $10 \%$ sodium dodecyl sulfate polyacrylamide gel electrophoresis and transferred to PVDF membranes. After being blocked with TBST buffer containing 5\% nonfat skim milk for $1 \mathrm{~h}$, the 
membranes were incubated overnight with primary antibodies against proliferating cell nuclear antigen (PACSIN2, 1:5000; Abcam, Cambridge, MA, USA), PKC (1:1000; Abcam), RCAN1.4 (1:1000), RCAN1.1 (1:1000) and GAPDH (1:10000; Cell signaling technology, Danvers, MA, USA) at $4{ }^{\circ} \mathrm{C}$. The membranes were then washed with TBST and incubated with a horseradish peroxidase-conjugated secondary antibody. The blots were developed using an Enhanced Chem luminescence Detection System (Thermo Scientific, Carlsbad, CA, USA)

\subsection{Statistical analysis}

All data was presented as mean \pm standard deviation (SD) and analyzed with the help of GraphPad Prism 7.0. The comparisons were performed with one-way ANOVA followed by Bonferroni post-hoc test. The statistical significance was defined as $P<0.05$.

\section{Results}

\subsection{Myocardial hypertrophy triggers changes in the expression level of PACSIN2}

In order to determine whether $\mathrm{MH}$ caused a change in the expression level of PACSIN2, based on a previous subject group study, and to determine the best time node for the model of $\mathrm{MH}$, this study used $1 \times 10^{\wedge}-6 \mathrm{M}$ All to stimulate primary cardiomyocytes at $0 \mathrm{~h}, 6 \mathrm{~h}, 12 \mathrm{~h}, 24 \mathrm{~h}$ and $48 \mathrm{~h}$. After All stimulation for $12 \mathrm{~h}$, it was noted that ANP mRNA significantly increased after $12 \mathrm{~h}$, BNP mRNA significantly increased after $6 \mathrm{~h}$, and PACSIN2 significantly increased after $3 \mathrm{~h}$ and was downregulated after $24 \mathrm{~h}$ (Figure 1A-C). It was discovered that, combined with bioinformatics research, PACSIN2 is correlated with PKC (Figure 1D). However, previous studies did not show that the PACSIN2 has a role in cardiovascular disease. Therefore, this study further analyzed the expression of PKC and PACSIN2 in primary cardiomyocytes under All stimulation. After $24 \mathrm{~h}$, the protein expression of PKC and PACSIN2 significantly decreased (Figure 1E), thereby indicating that there may be a positive correlation between PKC and PACSIN2.

\section{2 Up-regulation of PKC can promote the increase of PACSIN2 expression level}

For the purpose of further determining the relationship between PKC and PACSIN2 in MH, this study used the PKC agonist PMA to stimulate cardiomyocytes. A high PKC expression was elicited both in the hypertrophic state and with PMA stimulation, but it was reduced by All and PMA dual stimulation (Figure $2 \mathrm{~A}, 2 \mathrm{~B}$ ), and PACSIN2 protein was also largely consistent with the trend of PKC (Figure 2A, 2C). However, the expression of RCAN1.4 confirmed the hypertrophic features in the PMA, All, and All + PMA groups 
(Figure2A, 2D). In addition, this result was also reflected in HL1 cells. This shows that PKC certainly regulates the positive expression of PACSIN2 (Supplementary Figure 1).

\subsection{PKC causes morphological changes of cardiomyocytes}

In order to determine the pathological effects of acute PKC activation in myocardial hypertrophy, the study used the PKC agonist PMA as an inducing factor to analyze whether PKC activation changes the morphology of primary cardiomyocytes. Following the PMA stimulation of cardiomyocytes, they became morphologically bigger and presented a pathological hypertrophic morphology (Figure 3A-B). Meanwhile, hypertrophic features also appeared after an All stimulation of cardiomyocytes; however, without PMA stimulation, the hypertrophic morphology was prominent (Figure 3C). Meanwhile, upon dual stimulation with All and PMA, cardiomyocytes became morphologically smaller and developed features of heart failure (Figure 3D).

\subsection{Overexpression of PACSIN2 causes upregulation of $a-$ $\mathrm{MHC}$}

A PACSIN2 overexpression alone did not induce cardiac hypertrophy (Figure 4A-B); however, it caused an increase in the a-MHC expression and not in $\beta-\mathrm{MHC}$ (Figure 4C-D). In addition, since a-MHC is associated with microscopic proteins, the TUBULIN protein expression (Figure 4E) was examined; neither PACSIN2 overexpression nor knockdown had any effect on TUBULIN.

\section{Discussion}

In this study, it was initially found that PMA can stimulate cardiomyocytes to present the pathological $\mathrm{MH}$, whereas the state of heart failure appears under the hypertrophic cardiomyocytes. Interestingly, bioinformatics were used to discover a close association between PKC and PACSIN2. Then, it was further found that the expression of PACSIN2 in MH cell was increased, especially under the condition of PKC activation. An overexpression of PACSIN2 increased the expression of a-MHC in cardiomyocytes, but the expression of tubulin was not affected.

Previous studies have shown that PACSIN2 plays an important role in lipid trafficking and metabolism ${ }^{20}$ and participates in various biological activities, including various membrane deformations, such as behavioral reorganization, cystic transport and microtubule movement ${ }^{21}$. Although PACSIN2 is expressed in cardiomyocytes, there was no report of its specific function in cardiomyocytes. This study found that the expression of PACSIN2 in hypertrophic myocardial tissue was increased compared with that in normal cardiomyocytes, and prolonged myocardial hypertrophy caused the expression of PACSIN2 to be lower than that in normal cardiomyocytes. This indicates that PACSIN2 is related to the compensatory mechanism of the myocardium and the contraction of myocardial cells. Therefore, based 
on bioinformatics to explore PACSIN2 related regulatory factors, it is interesting that PKC and PACSIN2 were found to have a physical interaction relationship. Both classic and novel PKC are involved in the regulation of cardiac cell differentiation, proliferation and cardiac hypertrophy ${ }^{22}$, which provides a basis for exploring the regulation of cardiac hypertrophy by PACSIN2. When considered together, this data indicated that PACSIN2 participates in the maintenance and movement of cardiomyocytes.

Following that, the relationship between PKC and PACSIN2 in MH was further analyzed. Previous studies have shown that PKC can bind and phosphorylate PACSIN2 serine 313, thereby reducing its membrane binding and tubular capacity ${ }^{23}$. In addition, PKC phosphorylation of PACSIN2 plays a role in the breakage and stabilization of caveolae on the cell surface under hypotonic stress and shear stress ${ }^{24}$. This data indicates that PKC is the upstream signaling molecule of PACSIN2. In this study, activation of PKC can cause an upregulation of PACSIN2 expression in cardiomyocytes and hypertrophic cardiomyocytes. However, PMA stimulates the expression of PACSIN2 in hypertrophic cardiomyocytes to be lower than that in normal cardiomyocytes, which may be caused by the compensatory effect of hypertrophic cardiomyocytes. The activation of PKC further aggravates this process, ultimately leading to heart failure. These results indicate that PKC is an upstream signaling molecule of PACSIN2, and it aggravates the compensatory response of cardiomyocytes.

$\mathrm{MH}$ is a slower but more effective compensatory function. It mainly occurs in the case of a long-term pressure overload ${ }^{25}$. The total amount of myocardium is increased and the contractile force is strengthened so that the heart can maintain a normal blood circulation ${ }^{22}$. The results of the study indicate that a simple increase in PKC causes the production of cardiac hypertrophy and will further compound the hypertrophy compensatory effect. Therefore, PACSIN2, as a downstream molecule of PKC, can possibly play an important role in the contraction of cardiomyocytes. PACSIN2 plays a significant part in intracellular trafficking, thereby causing a signaling in many cell types. PACSIN2 Ko can reduce the efficiency of cardiac pacing and prolong $\mathrm{AV}$ conduction time in mice ${ }^{26}$, and $\mathrm{a}-\mathrm{MHC}$ is related to myocardial contractility ${ }^{27}$. This study has shown that an overexpression of PACSIN2 leads to the upregulation of a-MHC. This also confirms the hypothesis that PACSIN2 is involved in the myocardial contraction function.

\section{Conclusion}

PKC can be added to PACSIN2 to aggravate MH, and it is likely that PACSIN2 acts through Caused by aMHC. This study plays an important role in understanding the function of PACSIN2 and treating MH.

\section{Abbreviations}

MH: Myocardial hypertrophy; PKC: protein kinase C; All: angiotensin II; BAR: Bin-AmiphisinRvs; SD: standard deviation. 


\section{Declarations}

\section{Acknowledgements}

We would like to thank the technical support of Institute of Molecular Biology of Fudan University and all participants enrolled in the present study.

\section{Conflict of interest}

None.

\section{Authors' Contributions}

The contributions of the authors involved in this study are as follows: conception and design: ZW D; development of methodology: SA W, R X and ZW D; analysis and interpre tation of data: SA W, ZW D and $\mathrm{XH} L$ writing, review, and/or revision of the manuscript: SA W and YZ Z.

\section{Funding}

This work was supported by National Natural Science Foundation of China (No.: 81700256 and 81900245).

\section{Availability of data and materials}

The datasets generated/analyzed during the current study are available

\section{Ethics approval and consent to participate}

This study was carried out in compliance with the ARRIVE guidelines (http://arriveguidelines.org). all methods were carried out in accordance with relevant guidelines and regulations, and the study is approved by Beijing viewsolid biotechnology co.Itd animal experiment ethics committee, China.

\section{Consent for publication}

No applicable. 


\section{Competing interests}

The authors declare that they have no competing interests

\section{References}

1. Mishra S, et al. Cellular and molecular pathobiology of heart failure with preserved ejection fraction, Nature reviews. Cardiology. 2021;18:400-23.

2. Di Lullo L, et al. Chronic kidney disease and cardiovascular complications. Heart Fail Rev. 2015;20:259-72.

3. Ruddox $\mathrm{V}$, et al. Atrial fibrillation and the risk for myocardial infarction, all-cause mortality and heart failure: A systematic review and meta-analysis. Eur J Prev Cardiol. 2017;24:1555-66.

4. Yildiz M, Esenboğa K, Oktay AA. Hypertension and diabetes mellitus: highlights of a complex relationship. Curr Opin Cardiol. 2020;35:397-404.

5. Zhou H, et al. Mitophagy in cardiovascular disease. Clin Chim Acta. 2020;507:210-8.

6. Chelladurai $P$, et al. Targeting histone acetylation in pulmonary hypertension and right ventricular hypertrophy. Brit J Pharmacol. 2021;178:54-71.

7. Nakamura M, Sadoshima J. Mechanisms of physiological and pathological cardiac hypertrophy. Nat Rev. 2018;15:387-407.

8. Shimizu I, Minamino T. Physiological and pathological cardiac hypertrophy. J Mol Cell Cardiol. 2016;97:245-62.

9. Stylianidis V, et al. Wnt Signaling in Cardiac Remodeling and Heart Failure. Clin Cardiol. 2017;243:371-93.

10. Wang Y, Wang C, Ma J. Role of cardiac endothelial cells-derived microRNAs in cardiac remodeling. Discov Med. 2019;28:95-105.

11. Chitu V, Stanley ER. PACSIN2: a BAR-rier forming the megakaryocyte. DMS Blood. 2015;126:5-6.

12. Plomann $M$, et al. PACSIN, a brain protein that is upregulated upon differentiation into neuronal cells. Eur J Biochem. 1998;256:201-11.

13. Smid A, et al. Mlinaric-Rascan, PACSIN2 polymorphism is associated with thiopurine-induced hematological toxicity in children with acute lymphoblastic leukaemia undergoing maintenance therapy. Sci Rep. 2016;630:244.

14. Dumont $\mathrm{V}$, et al. PACSIN2 accelerates nephrin trafficking and is up-regulated in diabetic kidney disease. FASEB J. 2017;31:3978-90.

15. Sanderlin AG, et al. RNAi screen reveals a role for PACSIN2 and caveolins during bacterial cell-to-cell spread. Mol Biol Cell. 2019;30:2124-33. 
16. Gusmira A, et al. Regulation of caveolae through cholesterol-depletion-dependent tubulation mediated by PACSIN2, J. Cell. Sci 133, (2020).

17. de Kreuk B, et al. The F-BAR domain protein PACSIN2 associates with Rac1 and regulates cell spreading and migration. J Cell Sci. 2011;124:2375-88.

18. Malinova TS, et al. A junctional PACSIN2/EHD4/MICAL-L1 complex coordinates VE-cadherin trafficking for endothelial migration and angiogenesis. Nat Commun. 2021;12:2610.

19. Postema MM, et al. PACSIN2-dependent apical endocytosis regulates the morphology of epithelial microvilli. Mol Biol Cell. 2019;30:2515-26.

20. Matthaeus C, Taraska JW. Energy and dynamics of caveolae trafficking. Front Cell Dev Biol. 2020;8:614472.

21. Hubert M, Larsson E, Lundmark R. Keeping in touch with the membrane; protein- and lipid-mediated confinement of caveolae to the cell surface. Biochem Soc T. 2020;48:155-63.

22. Karhu ST, Ruskoaho H, Talman V. Distinct regulation of cardiac fibroblast proliferation and transdifferentiation by classical and novel protein kinase $\mathrm{C}$ isoforms: possible implications for new antifibrotic therapies. Mol Pharm. 2021;99:104-13.

23. Senju YS, Suetsugu. Possible regulation of caveolar endocytosis and flattening by phosphorylation of F-BAR domain protein PACSIN2/Syndapin II. Bioarchitecture. 2015;5:70-7.

24. Senju $Y$, et al. Phosphorylation of PACSIN2 by protein kinase $C$ triggers the removal of caveolae from the plasma membrane. J Cell Sci. 2015;128:2766-80.

25. Nakamura M, Sadoshima J. Mechanisms of physiological and pathological cardiac hypertrophy. Nat Rev Cardio. 2018;15:387-407.

26. Semmler $\mathrm{J}$, et al. Pacsin 2 is required for the maintenance of a normal cardiac function in the developing mouse heart. Pharmacol Res. 2018;128:200-10.

27. Palmer BM. Thick filament proteins and performance in human heart failure. Heart Fail Rev. 2005;10:187-97.

\section{Figures}


A

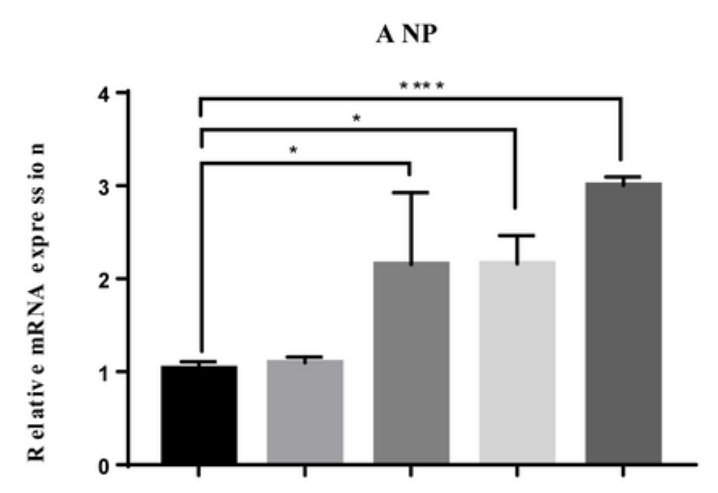

C

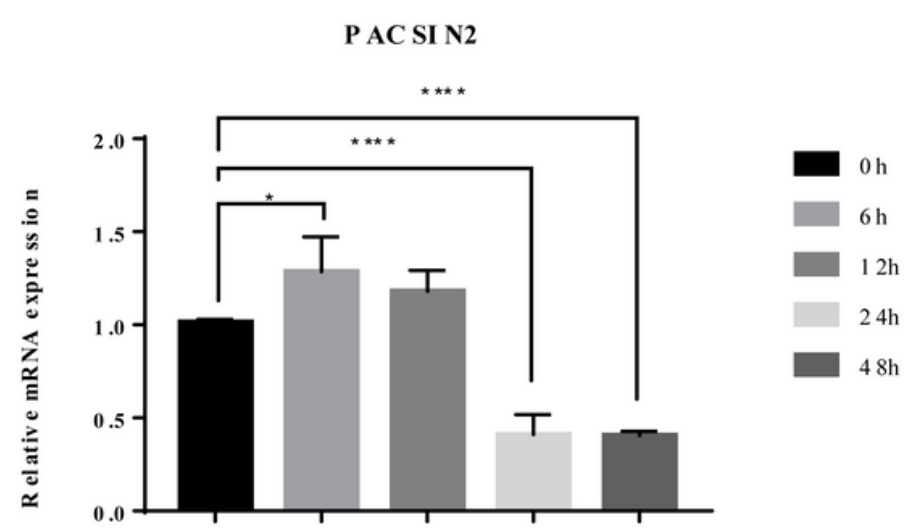

B

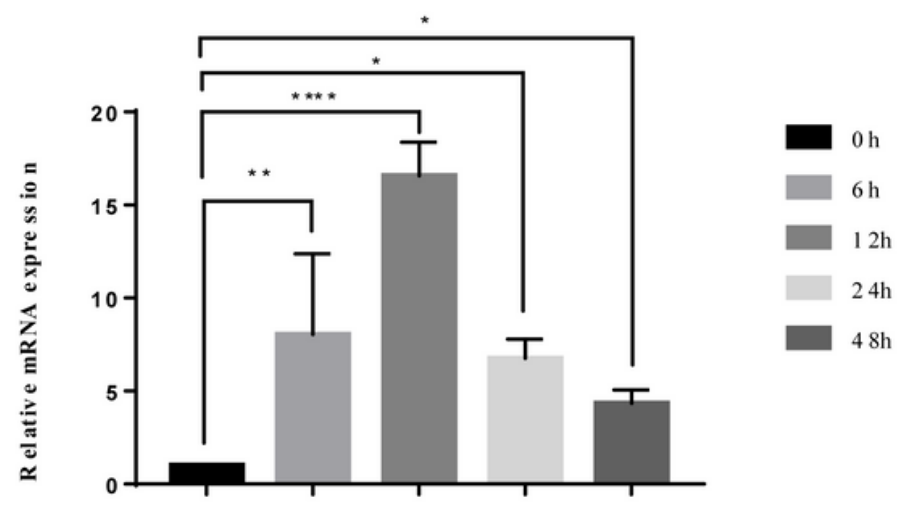

D

С M

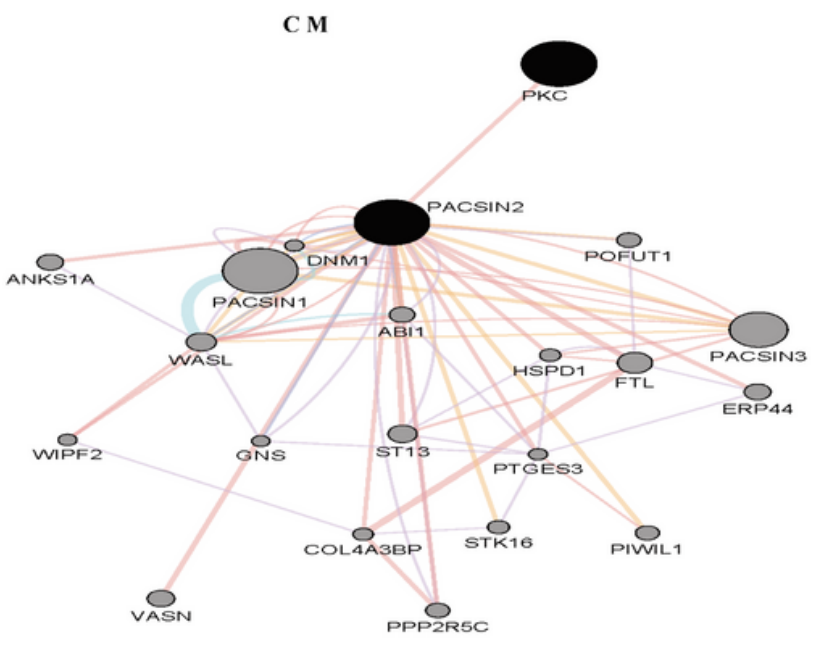

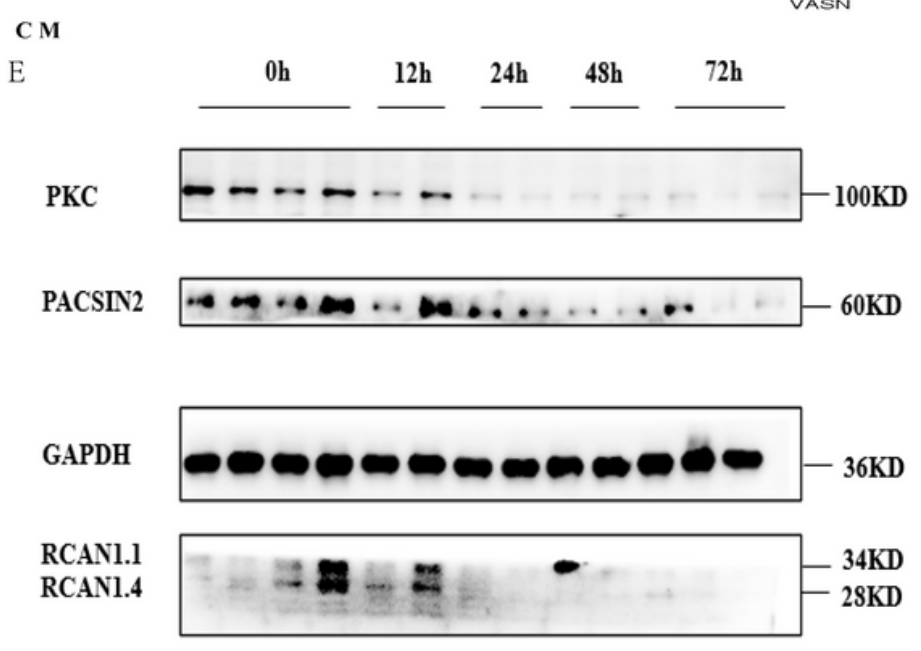

Figure 1

PACSIN2 expression changes induced by $\mathrm{MH}$. A-C: The mRNA expression of ANP, BNP and PACSIN2 were detected by qRT-PCR at 0 h, 6 h, 12 h, 24 h and 48 h after angiotensin II stimulation. D: Bioinformatics showed that PKC was correlated with PACSIN2. E: After stimulation CM at different time, the expression of PKC, PACSIN2, RCAN1.1 and RCAN1.4 were detected by Western blotting. N= 5, *P $<0.050$ h vs. 6 h, 12 h, 24 h, 48 h. Cardiac myocytes: CM. 
A

Contro

PMA

AII

AII + PMA

PKC

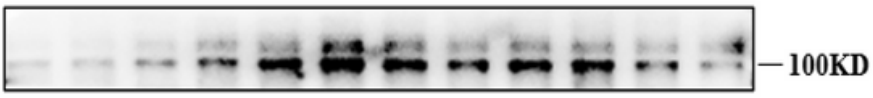

PACSIN2
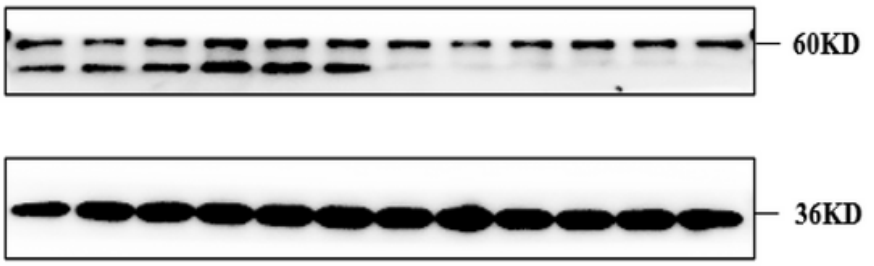

RCAN1.4

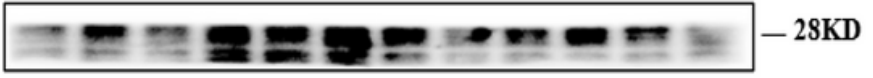

C

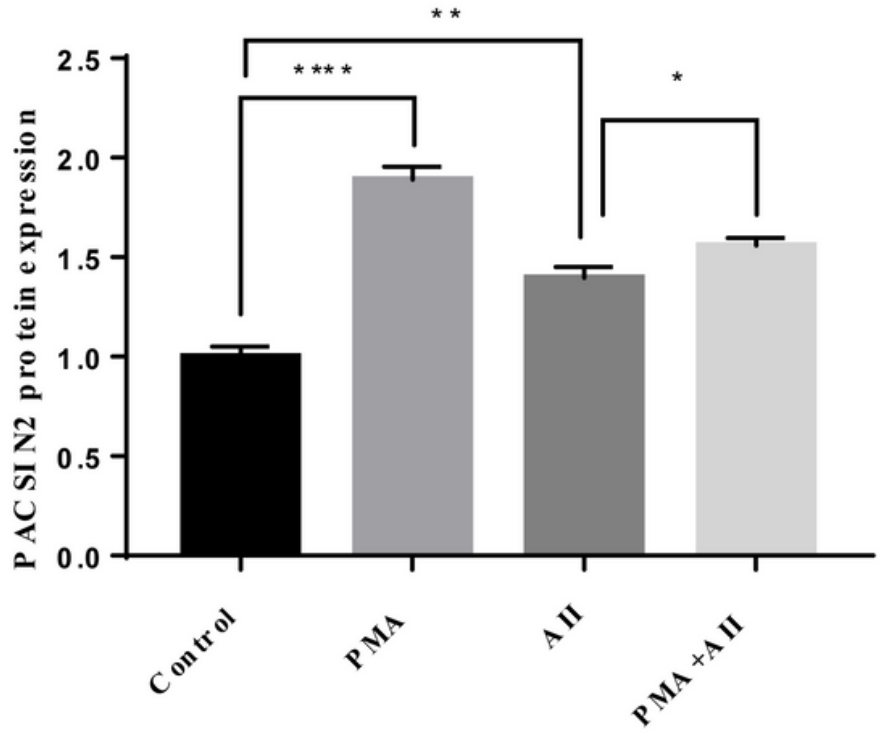

B

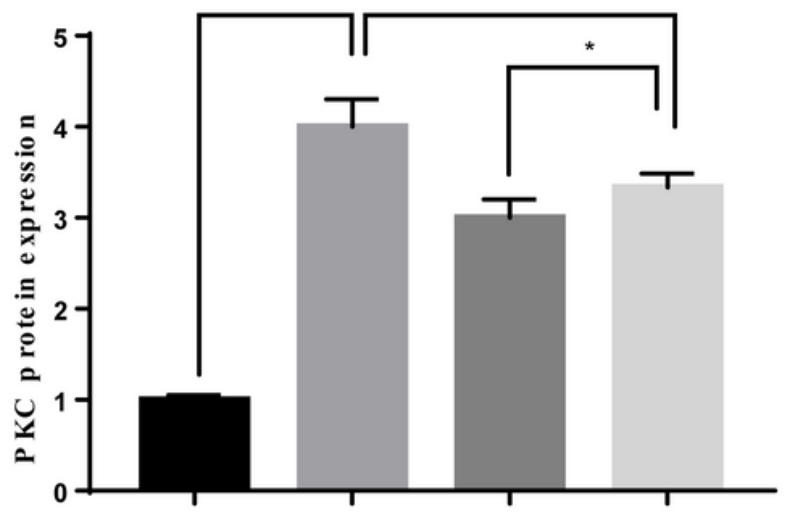

D
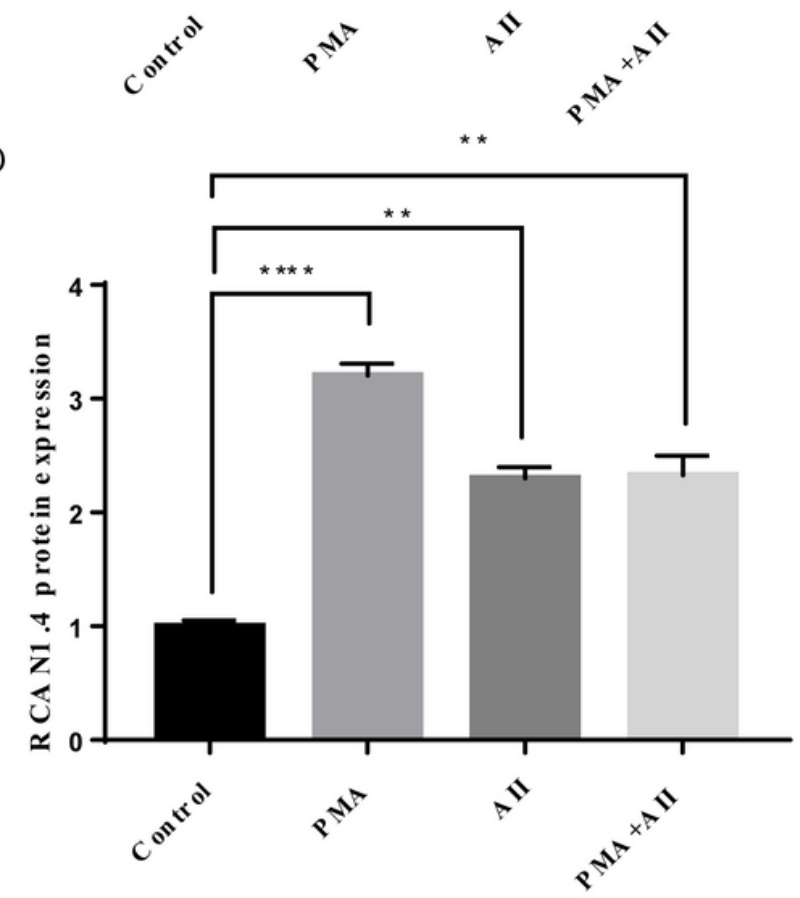

Figure 2

PKC regulates PACSIN2 expression with positive correlation. A-D: The expressions of PKC, PACSIN2 and RCAN1.4 in normal and hypertrophic cardiomyocytes were detected by Western blotting after PMA stimulation. Angiotensin II: All. ${ }^{*} P<0.05,{ }^{*} P<0.01,{ }^{*} * \star * P<0.0001$. 


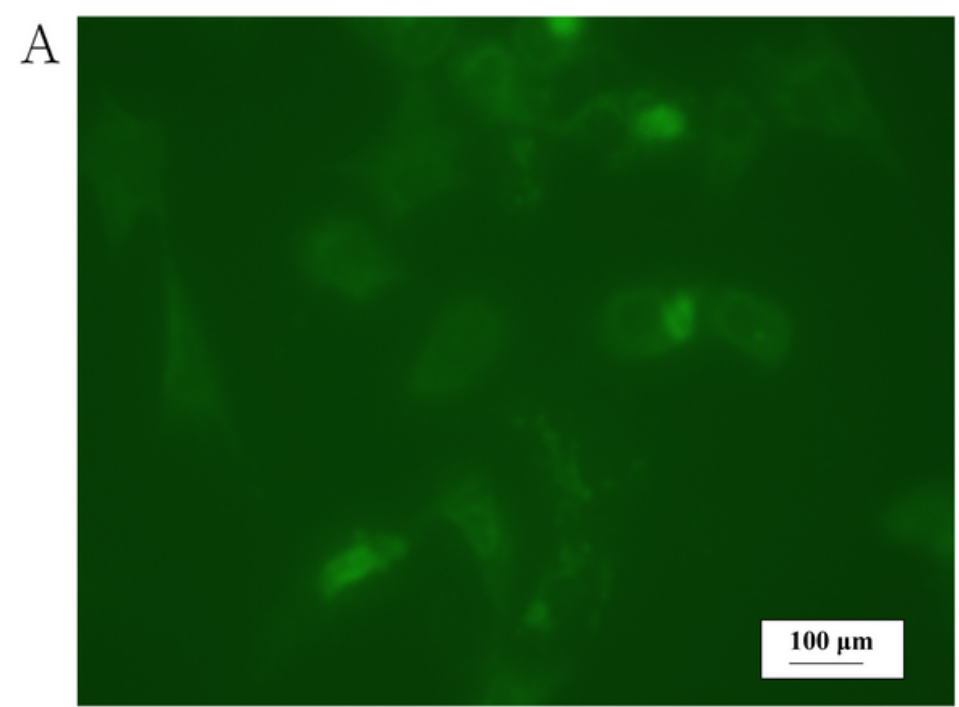

Control

C

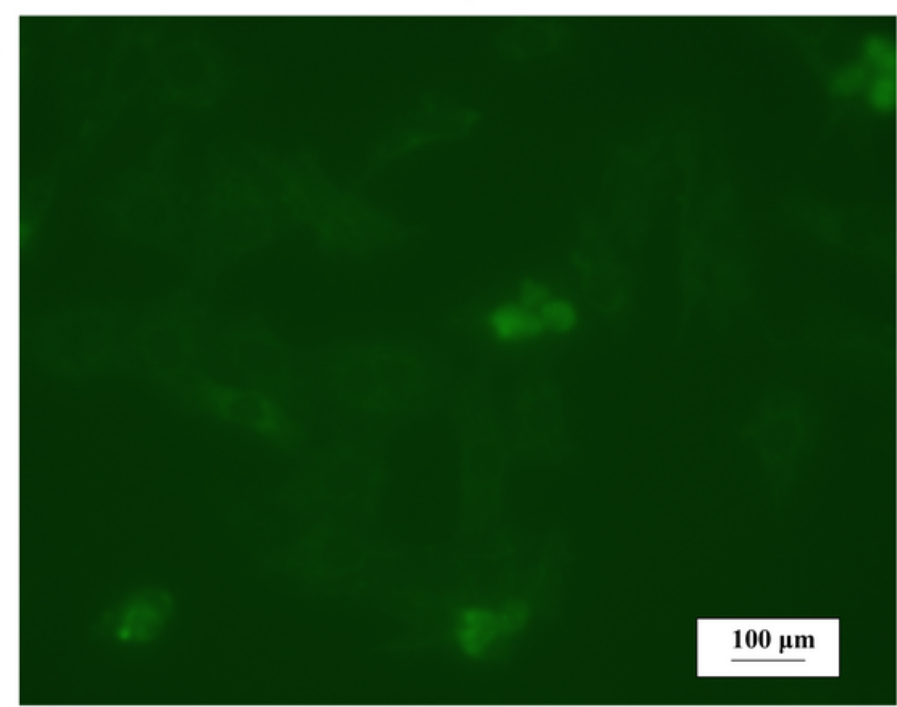

AII

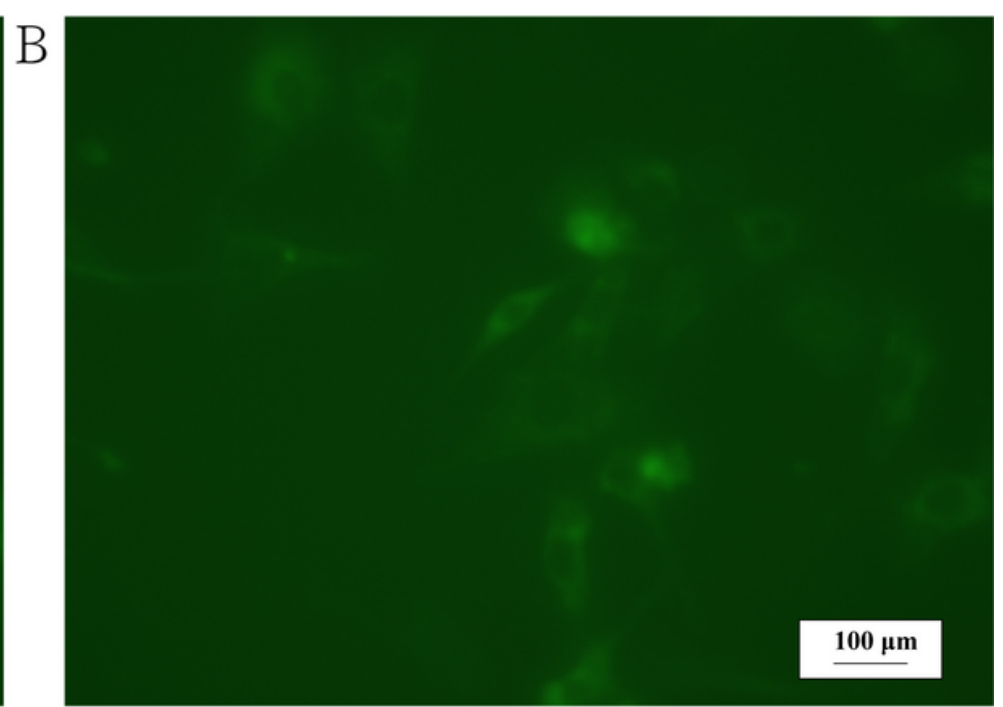

PMA

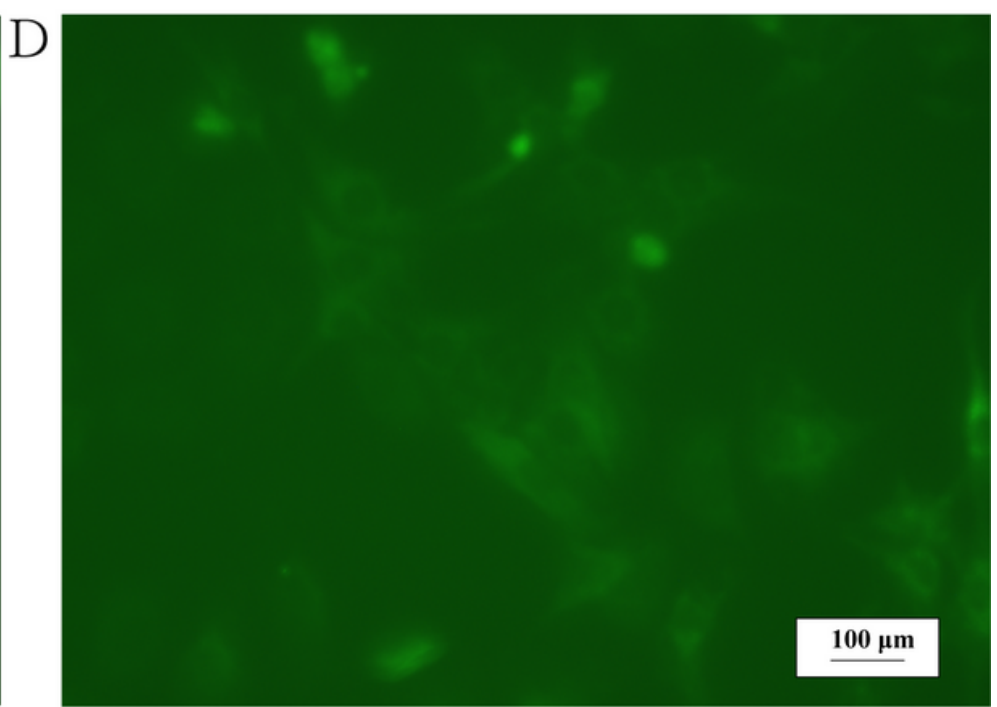

PMA+AII

\section{Figure 3}

morphological effects of PKC activation on hypertrophic cardiomyopathy. The differences between normal and hypertrophic cardiomyocytes among normal group, PMA group, All group, and PMA+All groups were evaluated by immunofluorescence experiments after PMA stimulation. 
A

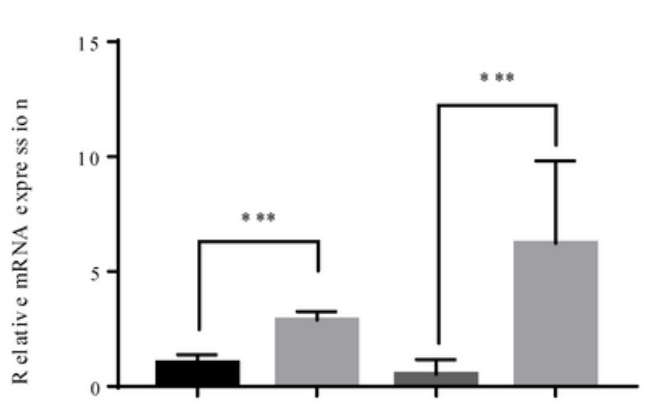

C

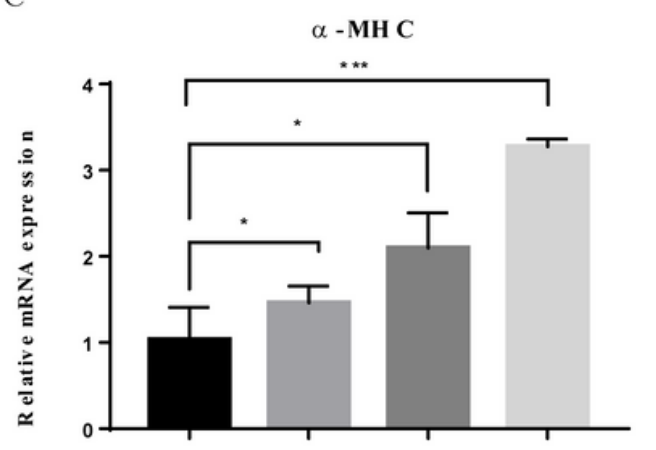

$\mathrm{CM}$
B

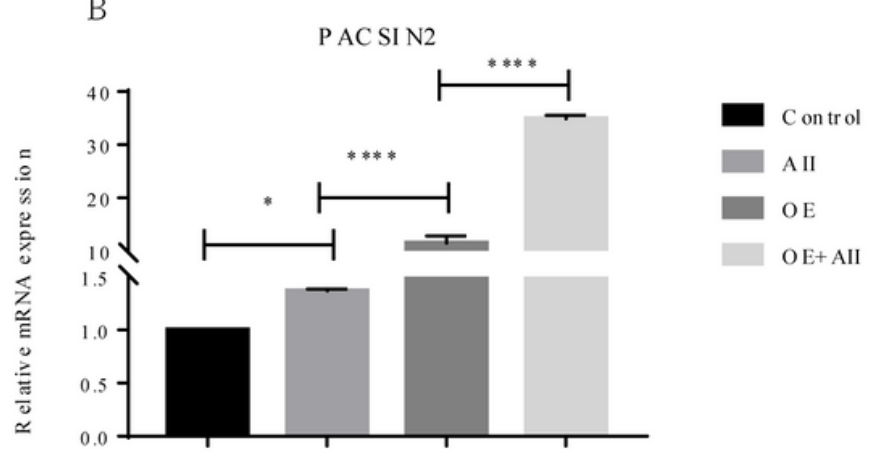

$\mathrm{CM}$

$\beta-\mathrm{MHC}$

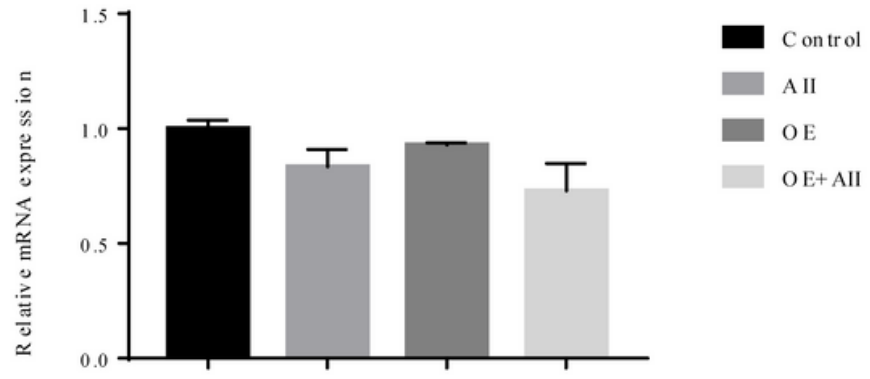

$\mathrm{C} M$

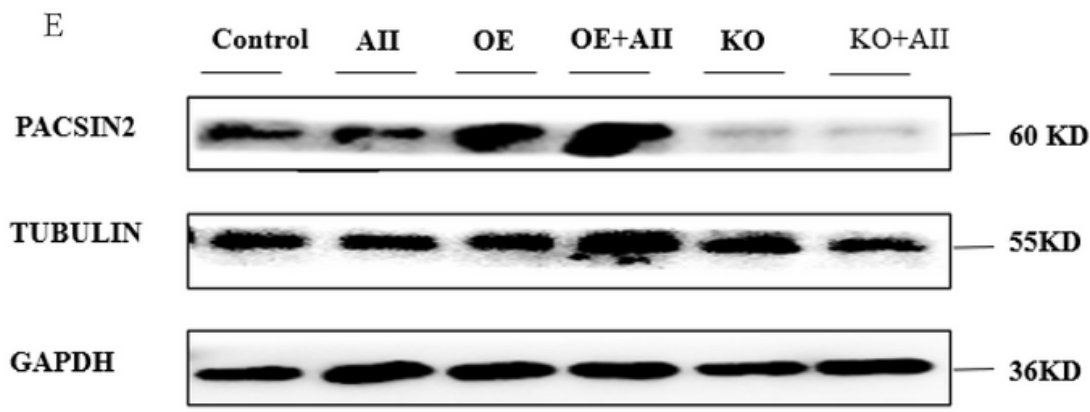

Figure 4

PACSIN2 is associated with cardiomyocyte contraction. A-D: After transfection with the PACSIN2 overexpression plasmid, BNP, PACSIN2, a-MHC, $\beta-\mathrm{MHC}$ mRNA expression. were determined by qRT-PCR. E: TUBULIN was assessed by Western blotting under overexpressed and silenced PACSIN2. Over expression: OE. *P<0.05, **P<0.01, **** $P<0.0001$.

\section{Supplementary Files}

This is a list of supplementary files associated with this preprint. Click to download.

- supplementarymaterial.docx

- WBdisplayedfigure.rar 